\title{
Identificação do nicho de progenitores mesenquimais no fígado de embriões e fetos caninos: uma fonte de células-tronco para terapia celular ${ }^{1}$
}

\author{
Daniele S. Martins ${ }^{2 *}$, Cristiane V. Wenceslau ${ }^{5}$, Eduardo Vieira ${ }^{3}$, André Luis R. Franciolli, \\ Carlos E. Ambrósio ${ }^{2}$, Ana F. Carvalho ${ }^{6}$ e Maria A. Miglino ${ }^{4}$
}

\begin{abstract}
Martins D.S., Wenceslau C.V., Vieira E., Franciolli A.L.R., Ambrósio C.E., Carvalho A.F. \& Miglino M.A. 2012. [Identification of mesenchymal progenitor niches from liver of embryo and fetuses of canines: a source of stem cells for cell therapy.] Identificação do nicho de progenitores mesenquimais no fígado de embriões e fetos caninos: uma fonte de células-tronco para terapia celular. Pesquisa Veterinária Brasileira 32(Supl.1):15-20. Departamento de Zootecnia, Faculdade de Zootecnia e Engenharia de Alimentos, USP, Av. Duque de Caxias Norte 225, Pirassununga, SP 13635-900, Brazil. E-mail: daniele@usp.br

Stem cells (SC) derived from fetal tissues (FT) were the most recent discoveries among the SC, and lately had demonstrated broad therapeutic potential; the FT from the fetal liver (FL) presents great therapeutic potential. This organ during the fetal period in mammals acts as a transient hematopoietic niche, being the main organ responsible for hematopoiesis in the fetus, and contribute to the formation of permanent niche in the adult bone marrow, thus can be considered a niche for mesenchymal stem cells (MSC) and parents. However, little is known about the location of these cells in FF, so the present study aims to identify the niche of mesenchymal progenitors in FF and dogs in order to contribute to the techniques of cell isolation and extraction. Together was performed to verify the expression of the transcription factor Oct-3/4 of the protein and DNA polymerase delta (PCNA). For the analysis of five embryos were used and 11 canine fetuses with gestational ages ranging from 25-60 days. The results elucidated from 25 days of gestation showed the FF is bulky and composed of all the typical structures, among them the portal triad, bile ducts and hepatic artery branches. With 30 days of gestation were identified the first requirements of mesenchymal progenitors (MP) at 60 days while the niches were completely formed with location similar to adult liver (AL). However, cells immunoreactives for Oct-3/4 were not identified, therefore, point out that the FL is a source of PM, presenting as an alternative for therapeutic purposes as well as for studying the developmental biology of MSC and parents.
\end{abstract}

INDEX-TERM: Liver, dogs, morphology, stem cells.

\footnotetext{
${ }^{1}$ Recebido em 16 de julho de 2012.

Aceito para publicação em 20 de setembro 2012.

${ }^{2}$ Faculdade de Zootecnia e Engenharia de Alimentos, Universidade de São Paulo (USP), Av. Duque de Caxias Norte 225, Pirassununga, SP $13635-$ 900, Brasil. *Autor para correspondência: daniele@usp.br

${ }^{3}$ Médico Veterinário autônomo, Centro Universitário da Fundação de Ensino Octávio Bastos, Avenida Dr. Octávio Bastos 2439, Jardim Nova São João, São João da Boa Vista, SP 13874-148, Brasil.

${ }^{4}$ Faculdade de Medicina Veterinária e Zootecnia, USP, Av. Prof. Dr. Orlando Marques de Paiva 87, Cidade Universitária, São Paulo, SP 05508-270, Brasil.

${ }^{5}$ Laboratório de Genética, Instituto Butantan, Av. Vital Brasil 1500, São Paulo, SP 05503-900.

${ }^{6}$ Centro Universitário da Fundação de Ensino Octávio Bastos, Av. Dr. Octávio Bastos s/n, Jardim Nova São João, São João da Boa Vista, SP, 13870000, Brasil.
}

RESUMO.- As células-tronco (CT) derivadas dos tecidos fetais (TF) foram as mais recentes descobertas entre as CT, e ultimamente tem demonstrado amplo potencial terapêutico, dentre os $\mathrm{TF}$ o fígado fetal $(\mathrm{FF})$ apresenta grande potencial terapêutico. Este órgão durante o período fetal em mamíferos é um nicho hematopoético transitório, sendo o principal órgão responsável pela hematopoese no feto, além de contribuir com a formação do nicho definitivo na medula óssea adulta, portanto pode ser considerado um nicho de células-tronco mesenquimais (CTM) e progenitores. No entanto, pouco se sabe sobre a localização destas células no FF, desta forma o presente estudo visa identificar o nicho de CTM e progenitores em FF de cães, a fim de contribuir com as técnicas de isolamento e extração celular. Em 
conjunto foi realizada a verificação da expressão do fator de transcrição Oct-3/4 e da proteína delta polimerase do DNA (PCNA). Para a análise foram utilizados cinco embriões e 11 fetos caninos com idades gestacionais variando de 25-60 dias. Os resultados elucidaram a partir de 25 dias de gestação o FF apresentou-se volumoso e composto por todas as estruturas típicas, dentre elas a tríade portal, ductos biliares e ramos das artérias hepáticas. Com 30 dias de gestação foram identificados os primeiros sitos de progenitores mesenquimais (PM) enquanto que aos 60 dias os nichos estavam completamente formados com localização semelhante ao fígado adulto (FA). No entanto, células imunopositivas para 0ct-3/4 não foram identificadas; sendo assim, destacamos que o FF é uma fonte de PM, apresentando-se como uma alternativa para a utilização terapêutica, bem como para os estudos da biologia do desenvolvimento das CTM e progenitores.

TERMOS DE INDEXAÇÃO: Fígado, caninos, morfologia, células-tronco.

\section{INTRODUÇÃO}

0 feto e os tecidos fetais (TF) apresentam-se como potencial fonte para os transplantes celulares, pois possuem menor risco e contaminação viral e/ou bacteriana quando comparado com as os tecidos adultos (TA), por residirem em um ambiente imunoprivelegiado dentro do útero. Além disso, a maioria das células fetais (CF) prolifera mais rapidamente, em meio de cultura, quando comparadas às células adultas (CA) e outra característica apresentada pelos TF é a ausência de questionamento ético quanto a sua utilização clínica em transplantes (Bruder et al. 1997, Abdulrazzak et al. 2010).

As primeiras células-tronco (CT) utilizadas em transplantes foram às células-tronco hematopoiéticas (CTH) para o tratamento de doenças neoplásicas, hematológicas ou não, doenças metabólicas e deficiências imunológicas. No entanto, sua utilização traz certas limitações como, por exemplo, a necessidade de testes de imunocompatibiliade entre o doador-receptor (Azevedo \& Ribeiro 2000), enquanto que as CT isoladas dos TF são imunogênicas, ampliando a sua utilização terapêutica (O'Donogue \& Fisk 2004). Células-tronco mesenquimais isoladas do FF demonstraram um grande potencial terapêutico no tratamento de imunodeficiências e desordens hematológicas (Touraine et al. 1993).

0 fígado fetal é excepcionalmente volumoso, ocupando um amplo espaço da cavidade abdominal (König \& Liebich 2004). Este é o principal sitio para o desenvolvimento de progenitores hematopoiéticos, atuando na diferenciação e maturação da linhagem hematopoética definitiva (Koury et al. 2002) e esta função é assumida pelo fígado fetal a partir do terço médio de gestação em mamíferos (Badylak 2002). Acredita-se que está função é promovida pelo nicho de CTM e seus progenitores presentes no fígado que regulam a hematopoese (Banks 1992). 0 fígado ao nascimento sofre uma mudança morfológica e funcional quanto ao aumento dos hepatócitos em número e volume, enquanto o número de CTH diminui e ocorre o predomínio de progenitores hepáticos, células hepáticas e epitelial biliar.
Os progenitores hepáticos no fígado adulto são responsáveis pela capacidade de regeneração por meio de progenitores, que se localizam nos canais de Hering e entre as pequenas ramificações biliares intra-hepáticas, alinhadas com os hepatócitos e com a linhagem de células epiteliais ductulares e na zona periductular/intraportal (Theise et al. 1999, Vessey \& Hall 2001).

Porém pouco se sabe sobre o nicho das células no fígado fetal em especial sobre as células-tronco mesenquimais (CTM), desta forma o presente estudo visa demonstrar a localização destas células no fígado fetal de cães em diferentes idades gestacionais correlacionando os dados com a organogenese do órgão. Assim, o estudo possibilitará a compreensão do mecanismo de formação do nicho hematopoético e sua função, propondo um novo modelo para o estudo da hematopoese fetal. Este conhecimento servirá como fonte para futuras pesquisas relacionadas à extração de CT e progenitores a partir do fígado que poderão ser essenciais para a compreensão do hematopoese fetal e adulta, sendo utilizadas para futuras pesquisas de terapia celular para o tratamento para doenças hematológicas em humanos e animais.

\section{MATERIAL E MÉTODOS}

O estudo foi conduzido após aprovação da Comissão de Bioética do Centro Universitário da Fundação de Ensino Octávio Bastos, São João da Boa Vista/SP.

Foram analisados 5 embriões com idades gestacionais de 2530 dias e 11 fetos com 35-60 dias de gestação de cães sem raça definida. As idades gestacionais foram estimadas através do método de Crown-Rump (CR) estabelecido por Evans \& Sack (1973) variando entre 25 a 60 dias de gestação (Quadro 1). Os embriões e fetos obtidos eram oriundos de campanhas de castração oferecidas pelos Centros de Controle de Zoonoses, localizados em São João da Boa Vista e em cidades vizinhas.

0 procedimento para a coleta dos fetos caninos seguiu o como o descrito por Martins et al. (2011) após coletado, o material os animais foram seccionados e coletou-se uma amostra do fígado, os fragmentos foram submersos em solução aquosa de paraformaldeído a 4\% em tampão sódio-fosfato (PBS) (Sigma, St. Louis, Mo., USA). Seguidamente foram desidratados com séries de alcoóis em

Quadro 1. Idade gestacional aproximada de embriões e fetos caninos sem raça definida

\begin{tabular}{cccc}
\hline Fêmea & Número de fetos & Dias de gestação & Crown-Rump (CR) \\
\hline $\mathbf{1}$ & 3 & 25 & $26 \mathrm{~mm}$ \\
$\mathbf{2}$ & 2 & 30 & $40 \mathrm{~mm}$ \\
$\mathbf{3}$ & 3 & 35 & $50 \mathrm{~mm}$ \\
$\mathbf{4}$ & 3 & 45 & $80 \mathrm{~mm}$ \\
$\mathbf{5}$ & 3 & 55 & $140 \mathrm{~mm}$ \\
$\mathbf{6}$ & 2 & 60 & $145 \mathrm{~mm}$
\end{tabular}

Quadro 2. Anticorpos utilizados para marcação imunohistoquímica do fígado embrionário e fetal de cães

\begin{tabular}{lcccc}
\hline Anticorpo & Empresa & Catálago & Diluição & Fixação \\
\hline $\begin{array}{c}\text { Oct-3/4 } \\
(\text { C-10) }\end{array}$ & $\begin{array}{c}\text { Santa Cruz Bio- } \\
\text { technology }\end{array}$ & sc-5279 & $1: 100$ & Paraformoldeído \\
$\begin{array}{c}\text { Vimentin } \\
\text { (0.N.602) }\end{array}$ & $\begin{array}{c}\text { Santa Cruz Bio- } \\
\text { technology }\end{array}$ & sc-73259 & $1: 300$ & Paraformoldeído \\
$\begin{array}{c}\text { PCNA } \\
\text { (PC10) }\end{array}$ & $\begin{array}{c}\text { Santa Cruz Bio- } \\
\text { technology }\end{array}$ & sc-56 & $1: 300$ & Paraformoldeído
\end{tabular}


concentrações crescentes (70\% a 100\%) e diafanizados em xilol, seguido de inclusão em paraplast (Paraplast Embedding Media-Paraplast Plus, Oxford Lab., USA). Posteriormente, secções de 4-5 micrômetros foram realizadas em micrótomo. Os cortes provenientes foram corados com HE - Hematoxilina-Eosina, para análise celular.

0 processamento de imuno-histoquímica baseou-se no método descrito por Vejlsted et al. (2005), no qual utilizamos anticorpo OCT3/4, Vimentina, e PCNA, de acordo com o quadro abaixo (Quadro 2)

\section{RESULTADOS E DISCUSSÃO}

Este estudo foi o primeiro a demonstrar o nicho de PM durante o desenvolvimento do fígado em cães, correlacio- nando com as características morfológicas do desenvolvimento. O modelo canino por apresentar semelhanças morfológicas bem como fisiológicas ao ser humano é considerado um excelente modelo para os estudos da biologia do desenvolvimento das CT (Hoorn et al. 2004), entretanto não há referencias de estudos prévios nesta espécie animal.

A estimativa da idade gestacional dos fígados embrionários e fetais caninos deu-se através do método de Crown-Rump (CR), como descrito por Noden \& Lahunta (1990) e Evans \& Sack (1973), entretanto nossos resultados discordam do referido por esses autores para embriões e fetos caninos, pois houve grande variação do tamanho nos dias 25, 30 e 35 de gestação, no qual demonstramos que
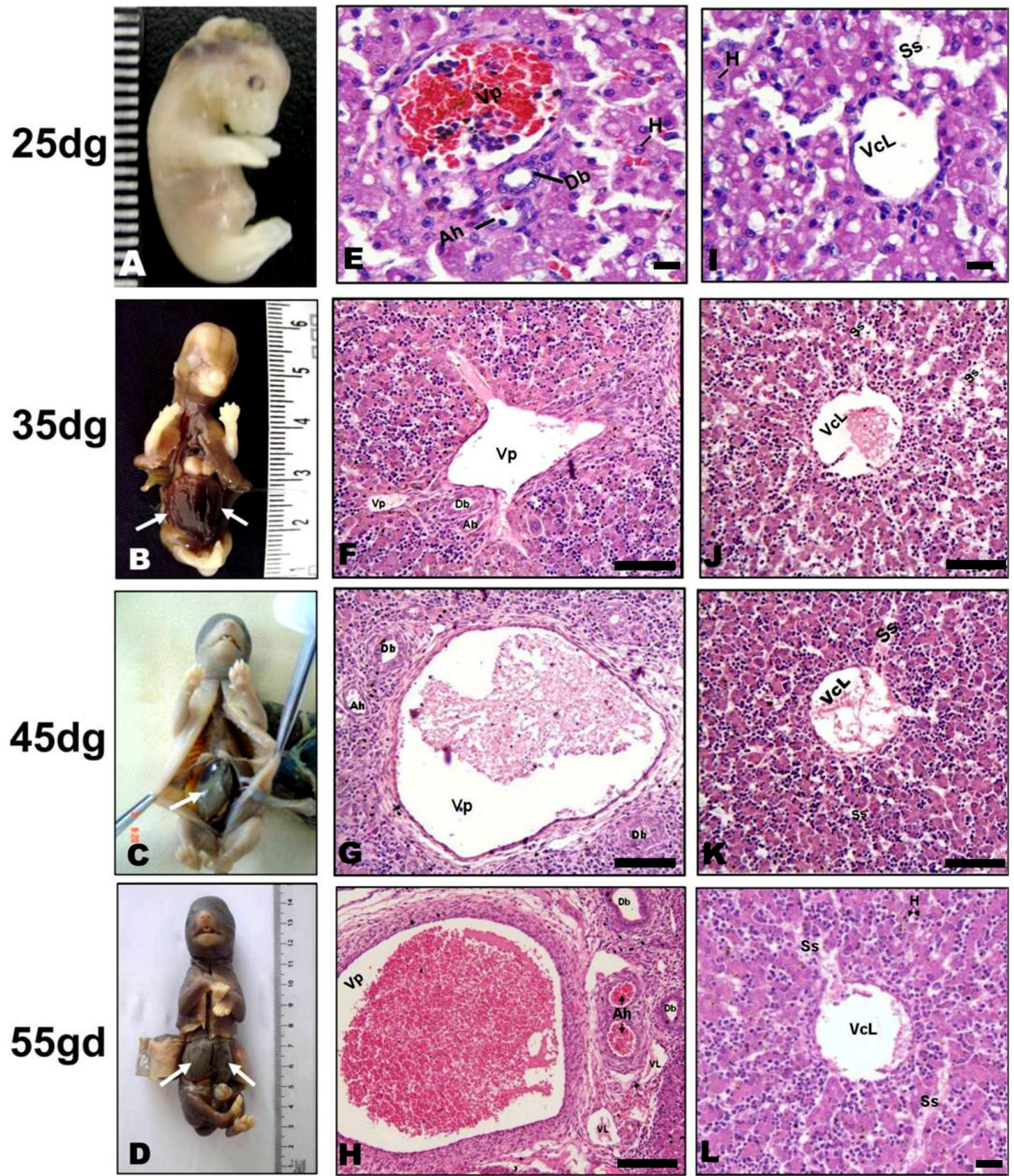

Fig.1. Estruturação do fígado fetal de cães SRD. (A, E e I) Fase gestacional de 25 dias. (B, F e J) Fase gestacional de 35 dias. (C, G e K) Fase gestacional de 45 dias. (D, H e L) Fetos de 55 dias de gestação. (A e B) Fígado fetal canino (setas). (E-H) Hepatócitos (H), veia porta (Vp), ducto biliar (Db) e ramos da artéria hepática (Ah). (I-L) Veia centro lobular (VcL), sinusoides (Ss) e hepatócitos (H). Coloração HE. Barras: $\mathbf{F}-\mathbf{H}, \mathbf{J}$ e $\mathbf{K}=100 \mu \mathrm{m}$ e $\mathbf{E}$, I e $\mathbf{L}=50 \mu \mathrm{m}$. 
os animais com essa idade apresentam aproximadamente o dobro do tamanho estipulado nestas literaturas. Todavia os períodos de 45, 55 e 60 dias de gestação o tamanho dos animais apresentou total confluência com nossos resultados.

Nossos resultados revelaram que os fígados fetais apresentaram-se extremamente volumosos em todas as faixas etárias estudadas, ocupando um amplo espaço da cavidade abdominal (Figura 1A-D) como descrito por Noden \& Lahunta (1990) e por König \& Liebich (2004), entretanto as analises histológicas demonstraram diferenças claras nas diferentes idades quando avaliamos a micro-organização do órgão.

Os dados histológicos elucidam que em todas as fases avaliadas o fígado fetal apresentou as estruturas típicas, composta pela presença da tríade portal, contendo a veia porta, ramos do sistema hepático biliar, representado pelos ductos biliares e ramos das artérias hepáticas (Fig.1E-H) assim como descrito por Ross et al. (2003) em vertebrados. Entretanto quando analisamos a veia centro lobular, destacamos que aos 25 dias de gestação à presença de hepatócitos imaturos com sinusoides venosos (Fig.1I), tal característica se mantém aos 35 e 45dias, todavia os hepatócitos apresentam-se em maior quantidade como o descrito por Lavon \& Benvenisty (2005) em camundongos, quando relata grande quantidade de hepatócitos em todas as idades gestacionais; outra característica demonstrada nesta fase gestacional é o processo de rearranjo celular indicando o inicio da formação dos cordões de hepatócitos (Fig.1J-L).

0 fígado fetal canino com 55 dias de gestação apresentou os hepatócitos dispostos ao redor da veia centrolobular em aglomerados celulares, formando placas celulares, as quais apresentaram-se direcionadas da periferia para o centro lobular, formando os sinusoides hepáticos (Fig.1L) como o sugerido por Godlewski et al. (1997) em ratos com 16 dias de gestação. Essas informações elucidam que o fígado canino sofre mudanças morfológicas e funcionais durante a ontogênese, demonstrada através reestruturação e organização dos hepatócitos em diferentes idades gestacionais, como o mencionado por Badylak (2002) para cães e Duncan (2003) para mamíferos.

Tal reestruturação e organização envolvem intensa atividade celular de proliferação. Esta atividade foi confirmada através da imunopositividade nos núcleos dos hepatócitos a proteína delta polimerase do DNA (PCNA), proteína auxiliar que tem papel essencial na replicação e reparação do DNA (Shivji et al. 1992). As analises de imunohistoquimica indicam que o fígado fetal está em constante atividade celular e que possivelmente é mediada através de células-tronco e progenitores celulares, os quais contribuem para o rápido crescimento do embrião e do feto (Fig.2E,F), como relatado por Harada et al. (1997) em humanos, Yoshioka et al. (2004) em cães, Suksaweang et al. (2004) em frangos e Banlunara et al. (2005) em patos.

A intensa atividade hepática não esta somente resumida ao crescimento hepático, mas também a função hematopoética que o fígado fetal exerce durante a ontogênese. Este órgão possui um ambiente que fornece condições a diferenciação e a maturação dos progenitores hematopoéticos em células adultas funcionais, que estão em contínua atividade celular.

A vimentina é uma proteína de citoesqueleto que se expressa em células de origem mesodermal, inclusive em CTM. Células imunopositivas para esta proteína foram observadas a partir de 30 dias de gestação no FF. Neste período gestacional a imupositividade foi verificada na cápsula hepática e em algumas células do parênquima hepático; en-
Oct $3 / 4$
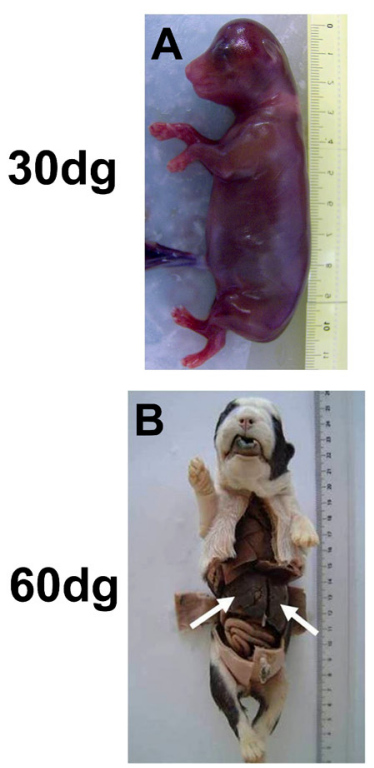
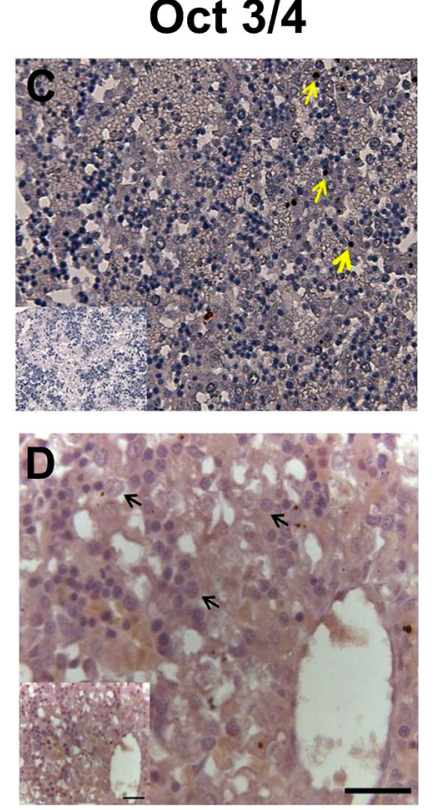

PCNA
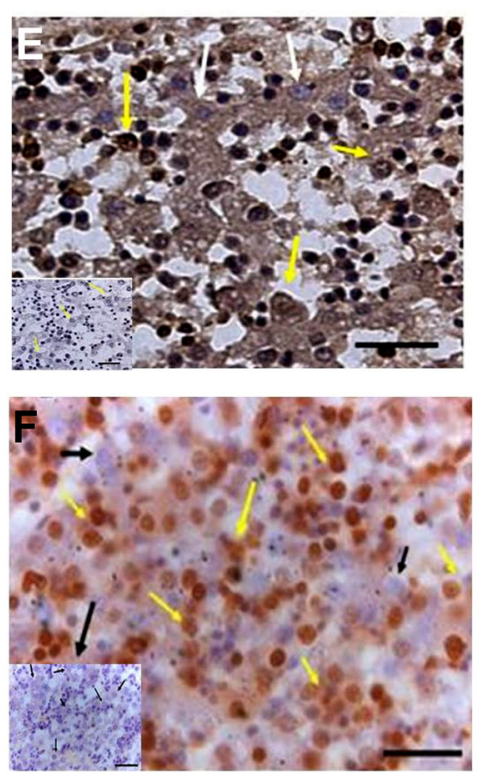
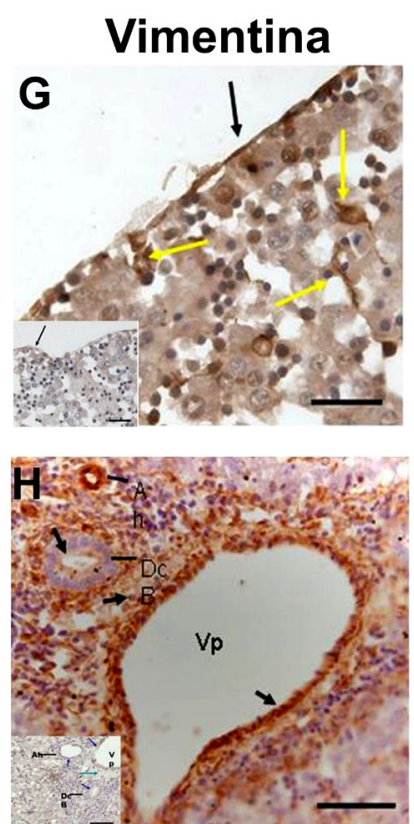

Fig.2. Imuno-histoquímica dos fígados fetais caninos com 30 e 60 dias de gestação. (A e B) Animais de 30 e 60 dias de gestação, respectivamente. (C e D) Núcleo negativo para o fator de transcrição Oct-3/4 (setas). (E e F) Imunopositividade do núcleo das células para PCNA (setas amarelas). (G) Pprogenitoras mesenquimais localizados na cápsula hepática e no parênquima (seta). (H) Imunopositividade das células ao redor da veia porta (vp), ducto biliar artéria hepática. Barras: $\mathbf{C}-\mathbf{F}=25 \mu \mathrm{m}, \mathbf{G}$ e $\mathbf{H}=10 \mu \mathrm{m}$. 
tretanto aos 60 dias de gestação era intensa a imunopositividade de células localizadas ao redor da tríade portal. Tais células no fígado fetal, contribuem para o nicho hematopoético transitório, emitindo sinalizações para as CTH controlando os mecanismos de diferenciação, apoptose e estado de quiescência das mesmas. (Fig.2G,H), indo ao encontro do descrito por Ijzer et al. (2006) em fígado fetal e/ou adulto. Em fígado adulto os progenitores hepáticos também se localizam preferencialmente próximos aos principais vasos e ductos do fígado, como o descrito por Theise et al. 1999, Vessey \& Hall 2001)

Os resultados obtidos neste estudo concordam com os dados colhidos por Campagnoli et al. (2001) e Mendes et al. (2009) que isolaram in vitro CTM a partir de fetos de humanos e camundongos, confirmando a utilização do fígado fetal como fonte de células tronco mesenquimais.

Entretanto, as analises imuno-histoquimicas revelaram que o fígado fetal não apresenta reatividade para o anticorpo Oct-3/4, que é um marcador específico para células-tronco embrionárias.(Figura 2C-D), diferente do descrito por Tai et al. (2005) em células-tronco de fígado humano, Pesce et al. (1998), Okamoto et al. (1990), Pesce \& Schöler (2001), Lengner et al. (2007) e Schöler et al. (1990) em camundongos, e Tai et al. (2005) em humanos.

\section{CONCLUSÃO}

Os resultados demonstraram que progenitoras mesenquimais podem ser identificadas no fígado fetal canino a partir de 30 dias de gestação, sendo que seu número progride proporcionalmente com o desenvolvimento e organização hepática. Assim como no fígado adulto, o nicho do fígado fetal localiza-se preferencialmente próximo a tríade portal. Sendo assim, este estudo contribui para as pesquisas de biologia do desenvolvimento das células tronco e seus progenitores, que contribuirão para o desenvolvimento de técnicas de isolamento in vitro destas células e de células tronco hematopoiéticas e seus progenitores.

Agradecimentos.- À Fundação de Amparo a Pesquisa do Estado de São Paulo (FAPESP) pelo apoio financeiro.

\section{REFERÊNCIAS}

Abdulrazzak H., Moschidou D., Jones G. \& Guillot P.V. 2010. Biological characteristics of stem cells from foetal, cord blood and extraembryonic tissues. J. Royal Soc. Interface 7:S689-S706.

Azevedo W. \& Ribeiro M.C. 2000. Fontes de células-tronco hematopoéticas para transplantes. Medicina, Ribeirão Preto, 33:381-389.

Badylak S.F. 2002. The extracellular matrix as a scaffold for tissue reconstruction. Seminars Cell Dev. Biol. 13:377-383.

Banks W.J. 1992. Histologia Veterinária Aplicada. $2^{a}$ ed. Manole, São Paulo, p.468-476.

Banlunara W., Bintvihok A. \& Kumagai S. 2005. Immunohistochemical study of proliferating cell nuclear antigen (PCNA) in duckling liver fed with aflatoxin B1 and esterified glucomannan. Toxicon 46(8):954-957.

Bruder S.P., Jaiswal N. \& Haynesworth S.E. 1997. Growth kinetics, self-renewal, and the osteogenic potential of purified human mesenchymal stem cells during extensive subcultivation and following cryopreservation. J. Cell Biochem. 64:278-294.

Campagnoli C., Roberts I.A., Kumar S., Bennett P.R., Bellantuono I. \& Fisk N.M. 2001. Identification of mesenchymal stem/progenitor cells in hu- man first-trimester fetal blood, liver, and bone marrow. Blood 98:23962402.

Duncan S.A. 2003. Mechanisms controlling early development of the liver. Mechanisms of Development 120(1):19-33.

Evans H.E. \& Sack W.O. 1973. Prenatal development of domestic and laboratory mammals: Growth curves, external features and selected references. Anat., Histol., Embryol. 2:11-45.

Fine A. 1994. Transplantation of fetal cells and tissue: An overview. Can. Med. Assoc. J. 15(9):1261-1268.

Gasper P.W. 2000. The hemopoietic system, p.63-68. In: Feldman B.F. (Ed.), Schalm's Veterinary Hematology. Williams and Wilkins, Philadelphia.

Godlewski G., Gaubert-Cristol R., Rouy S. \& Prudhomme M. 1997. Liver development in the rat and in man during the embryonic period (carnegie stages 11-23). Microsc. Res. Tech. 39(4):314-327.

Harada H., Imamura H., Miyagawa S. \& Kawasaki S. 1997. Fate of the human liver after hemihepatic portal vein embolization: Cell kinetic and morphometric study. Hepatology 26(5):1162-70.

Hoorn P.A., Morris J.C., Neff T. \& Kiem H.P. 2004. Stem cell gene transferefficacy and safety in large animal studies. Molecular Therapy 10:417443.

Ijzer J., Roskams T., Molenbeek R.F., Ultee T., Penning L.C., Rothuizen J. \& Van den Ingh T.S. 2006. Morphological characterisation of portal myofibroblasts and hepatic stellate cells in the normal dog liver. Comp. Hepatol. 16:5:7

König H.E. \& Liebich H.G. 2004. Anatomia dos Animais Domésticos: texto e atlas colorido. Artmed, Porto Alegre. 400p.

Koury M.J., Sawyer S.T. \& Brandt S.J. 2002. New insights into erythropoiesis. Curr. Opin. 9:93-100.

Lavon N. \& Benvenisty N. 2005. Study of hepatocyte differentiation using embryonic stem cells. J. Cell. Biochem. 96:1193-1202.

Lengner C.J., Camargo F.D., Hochedlinger K., Welstead G.G., Zaidi S., Gokhale S., Scholer H.R., Alexey Tomilin A. \& Jaenisch R. 2007. Oct4 expression is not required for mouse somatic stem cell selfrenewal. Cell Stem Cell 11(4):403-415.

Martins D., Ambrósio C., Saraiva N., Wenceslau C., Morini A., Kerkis I., Garcia J. \& Miglino M. 2011. Early development and putative primordial germ cells characterization in dogs. Reprod. Domest. Anim. 46(1):62-66.

Mendes S.C., Robin C. \& Dzierzak E. 2005. Mesenchymal progenitor cells localize within hematopoietic sites throughout ontogeny. Development and Disease 132:1127-1135.

Noden D.M. \& Lahunta A. 1990. Embriologia de los Animales Domésticos. Acriba, Zaragoza. 422p.

O’Donoghue K. \& Fisk N.M. 2004. Fetal stem cells. Brit. J. Obstetr. Gynaecol. 18:853-875.

Okamoto K., Okazawa H., Okuda A., Sakai M., Muramatsu M. \& Hamada H. 1990. A novel octamer binding trascription factor is differentially expressed in mouse embryonic cells. Cell 60:461-472.

Pesce M. \& Schöler H.R. 2001. OCT-4: Gatekeepe inthe beginning of mammalian development. Stem Cells 19:271-278.

Pesce M., Gross M.K. \& Schöler H.R. 1998. In line with our ancestors: Oct-4 and the mammalian germ. Bioessays 20:722-732.

Ross M., Kaye G. \& Pawlina W. 2003. Histología Texto y Atlas Color con Biología Celular y Molecular. 4ª ed. Panamericana, Buenos Aires. 924p.

Schöler H.R., Dressler G.R., Balling R., Rohdewohld H. \& Gruss P. 1990. Oct-4: A germline-specific transcription factor mapping to the mouse t-complex.A germline-specific transcription factor mapping to the mouse t-complex. Embo J. 9(7):2185-95.

Shivji K.K., Kenny M.K. \& Wood R.D. 1992. Proliferating cell nuclear antigen is required for DNA excision repair. Cell 69:367-374.

Suksaweang S., Lin C.M., Jiang T.X., Hughes M.W., Widelitz R.B. \& Chuong C.M. 2004. Morphogenesis of chicken liver: Identification of localized growth zones and the role of beta-catenin/Wnt in size regulation. Dev. Biol. 266(1):109-122.

Tai M.H., Chang C.C., Kiupel M., Webster J.D., Olson L.K. \& Trosko J.E. 2005. 
Oct-4 expression in adult human stem cells: evidence in support of the stem cell theory of carcinogenesis. Carcinogenesis 26:495-502.

Theise N.D., Saxena R., Portmann B.C., Thung S.N., Yee H., Chiriboga L., Kumar A. \& Crawford J.M. 1999. The canals of Hering and hepatic stem cells in humans. Hepatology 30:1425-1433.

Touraine R.L., Bertrand Y., Foray P., Gilly J. \& Philippe N. 1993. Hepatic tumours during androgen therapy in Fanconi anaemia. Eur. J. Pediatr. 152(8):691-693.
Vejlsted M., Avery B., Schmidt M., Greve T., Alexopoulos N. \& Maddox-Hyttel P. 2005. Ultrastructural and immunohistochemical characterization of the bovine epiblast. Biol. Reprod. 72:678-686.

Vessey C.J. \& Hall P.M. 2010. Hepatic stem cells: A review. Pathology 33:130-141.

Yoshioka K., Enaga S., Taniguchi K., Fukushima U., Uechi M. \& Mutoh K. 2004. Morphological characterization of ductular reactions in canine liver disease. J. Comp. Pathol. 130(2/3):92-98. 